\title{
New Score to Diagnose Covid 19 Infection Using Lung Ultrasound
}

Mohammad El Tahlawi ${ }^{1}$, hisham samir ${ }^{2}$, Mohammad Walaa ${ }^{1}$, Panagiota Manthou ${ }^{3}$, Diego Arazia-Garaygordobil ${ }^{4}$, Mohammad Elshabrawy ${ }^{1}$, Mohamed Elkholy ${ }^{5}$, and Waleed Mansour $^{1}$

${ }^{1}$ Zagazig University Faculty of Human Medicine

${ }^{2}$ Affiliation not available

${ }^{3}$ National and Kapodistrian University of Athens

${ }^{4}$ Instituto Nacional de Cardiologia Ignacio Chavez

${ }^{5}$ Zagazig University Faculty of Agriculture

May 6, 2020

\begin{abstract}
Background: PCR used for SARS-CoV-2 diagnosis is variable and unstable. Lung ultrasound has good safety and accuracy in pneumonia. We aim to evaluate the ability of bedside lung ultrasound in suspecting the diagnosis and/or predicting the prognosis of Covid 19 infection. Patients \& Methods: We recruited any case with pulmonary symptoms necessitating admission in intermediate care and/or ICU during the period between 27th March and 11th April 2020 in 3 international centres. Patients were stratified according to Covid 19 PCR results. All patients underwent chest X ray, CT, lung ultrasound, echocardiography and laboratory analysis. Results: Eighty-two cases were recruited. Fifty cases (61\%) were negative for SARS-CoV-2 while 32 cases (39\%) were positive. Sixty-four cases (78\%) were recovered while 18 cases (22\%) died. Covid 19 patients had more A profile and more abnormal A lines than non-Covid 19 group $(\mathrm{p}<0.001)$. Smoking, congestion in X ray, A profile and abnormal A lines in lung ULS are independent predictors for Covid 19 infection. A scoring system for prediction of Covid 19 using clinical and radiological data was calculated with a sensitivity of $93.8 \%$ and a specificity of $58 \%$. Mortality in Covid 19 patients is significantly correlated with age, duration of fever, presence of cardiac disease, B profile and abnormal A lines by lung ULS. However, it is negatively correlated with initial O2 saturation and EF by echocardiography Conclusion: Lung ULS could predict SARS-CoV-2 infection among patients with pulmonary symptoms necessitating admission. Lung ULS can also predict the outcome of Covid 19 infected patients.
\end{abstract}

\section{Introduction:}

Covid 19 (SARS-CoV-2) pandemic is the most serious medical problem worldwide nowadays.

One of the primary findings for COVID-19 is pneumonia (1)(2).

Many cases with chest infection may have similar symptoms and signs with SARS-CoV-2 infection.

Real-time reverse-transcriptase polymerase chain reaction (RT-PCR) assay is the test widely used to diagnose SARS-CoV-2 (3).

However, RT-PCR test results of pharyngeal swab specimens have some variability and potential instability, therefore it should not be considered as the only indicator for diagnosis (4)

Although Covid 19 infected patients have some typical radiological CT findings even in asymptomatic patients (5), atypical findings are increasingly common. Uptill now the role of CT poorly defined for diagnosis of 
Covid-19 patients or in expecting their outcome (6).

Few studies have reported the predictors of prognosis in Covid 19 patients (7)(8).

Lung ultrasound (ULS) has been widely considered over the last years due to its medical and surgical value (9)(10).

The role of lung ULS in the care of patients with critical respiratory conditions is nowadays widely documented (11) .

Aim: We aim to find simple bedside investigations that could suspect the diagnosis and/or predict the prognosis of Covid 19 infection.

\section{Patients $\mathcal{E}$ Metods}

\section{Inclusion criteria:}

- The study recruited any hospitalized case with pulmonary symptoms necessitating admission in intermediate care and/or ICU during the period between $27^{\text {th }}$ March and $11^{\text {th }}$ April 2020.

The study is international multicentre study included 3 centres (Cardiology and Chest hospital of Zagazig university hospitals and its isolation unit, Egypt), (Covid assigned Hospital, Athens ,Greece) and (National Institute of Cardiology, México)

* Exclusion criteria: Patients with one or more of the following criteria were excluded from the study: Patients with congestive heart failure - Patients with pulmonary embolism - Patients with poor echo-window

Patients were seen at first at Emergency Department (ED) that did the required investigations for all patients and classified them. Patients were first questioned about symptoms suspecting Covid 19 infection. Those who met the suspected clinical and investigational criteria were given a standard mask and were rapidly transferred in a safe way to a separate waiting and isolation area (isolation) with available infrastructure and tools for hand and respiratory hygiene practice. If the patient was proved to be positive for SARS-CoV-2, he was sent to quarantine. For negative patients, they were admitted to intermediate or ICU according to their clinical status.

\section{All recruited patients underwent the following:}

1. Chest X

2. CT chest

3. Complete blood count (CBC)

4. Arterial blood gas (ABG)

5. RT-PCR assay to detect SARS-CoV-2.

6. Lung ultrasound (LUS): with the following precautions

Two trained medical personnels, one ICU physician and one ICU nurse, entered the isolation room respecting all the preventive measures for respiratory, droplet and contact isolation provided by the World Health Organization for the nCoV-19 outbreak (12). The ultrasound probe and the tablet were put in two different sterile, plastic probe and tablet covers.

Imaging was performed using a curvilinear probe $(2-5 \mathrm{MHz})$ with different devices according to the availability in each center. Six-point lung ULS (three in each hemithorax) was performed as described in the BLUE protocol ( $B$ edside $L$ ung $U$ ltrasonography in $E$ mergency) (13).

Echocardiography : according to its availability, with precautions for the operators and for the probe similar to those exerted to lung ULS.

\section{Statistical analysis:}

Statistical analysis included comparing different parameters between patients with pneumonia and COVID19 positive and those with COVID19 negative test results, using independent t-test for numerical variables and 
chi-square for categorical variables. All significantly different variables were entered in a forward stepwise binary logistic regression analysis to select the best model. After selecting the best model. The variable chosen in the last step was weighed using the odds ratios calculated from the regression coefficient $(\beta)$ for each variable, the odds ratios were multiplied by 0.125 to calculate a score for each variable and the number was rounded to the nearest integer giving of scoring system of 10 points. All study group patients were scored. The cutoff point of the score was calculated using ROC analysis., and calculation of sensitivity, specificity and accuracy was performed. Also, variables associated with mortality in COVID19 positive were entered in a forward binary logistic regression, which selected the best model and the odds ratios was calculated for each variable using the regression coefficient $(\beta)$. Before entering the variables in the regression analysis determination of the proper cutoff values of different contentious variables was done using ROC analysis. patients Data were entered checked and analyzed using SPSS for Windows version 16 (SPSS, Inc. Chicago, IL, USA). For all the above mentioned statistical tests, the threshold of significance is fixed at $5 \%$ level (P $<0.05)$.

Results:

Eighty-two cases were recruited, 50 cases were males and 32 were females. Their mean age was $57.2 \pm 15.23$ years. Fifty cases $(61 \%)$ were negative for SARS-CoV-2 while 32 cases $(39 \%)$ were positive.

The mean hospital stay in days were $14+-4.14$ days. Sixty-four cases $(78 \%)$ were recovered and discharged home while 18 cases (22\%) died within 2 weeks of follow up.

There was significant difference between Covid 19 and non Covid patients regarding smoking $(\mathrm{p}=0.01)$ while there was no significant difference between both groups regarding sex, diabetes, hypertension or cardiac disease (table 1).

Regarding radiological examination, there was a high significant difference between both groups in the context of lung congestion in $\mathrm{X}$ ray with more congestion profile in Covid 19 patients $(\mathrm{p}=0.0004)$. In $\mathrm{CT}$ chest, there was more consolidation and ground glass appearance among Covid 19 patients $(\mathrm{p}<0.001)$ for each, while they had less pleural effusion than Covid 19 negative patients $(\mathrm{p}=0.026)$.

Concerning lung ULS, there was more A profile among Covid 19 group and more abnormal A lines among them with statistically significant difference between both groups $(\mathrm{p}<0.001)$ for both items $($ table 2$)$.

-Linear regression analysis for the independent predictors for being Covid 19 positive was done (table 3). Smoking, congestion in x ray,A profile and abnormal A lines in lung ULS are independent predictors for Covid 19 infection.

-A scoring system for prediction of Covid 19 diagnosis using clinical and radiological data was calculated (table 4). Score cutoff value was determined to $=5$ with a sensitivity of $93.8 \%$ and a specificity of $58 \%$ in detecting COVID19 positive patients (Figure 1)\& (table 5).

By performing the correlation between clinical, laboratory and radiological factors in Covid 19 positive patients on one side and mortality and duration of hospital stay on the other side, we found the following (table 6):

-There was significant positive correlation between mortality and each of age, duration of fever, presence of cardiac disease including hypertension, presence of B profile and abnormal A lines by lung ULS.

- There was significant negative correlation between mortality and each of initial O2 saturation and EF by echocardiography.

-There was significant positive correlation between duration of hospital stay and abnormal A lines.

Discussion:

Diagnosis of SARS-CoV-2 is done by RTPCR testing. However, there is limited availability and high cost of RTPCR testing for SARS-CoV-2, in addition to its potentially high false negative rate of patients clinically 
diagnosed with COVID-19. Furthermore, the RT-PCR results

showed a fluctuating trend (4). The timely transfer patients to ICU or a designated unit (isolation or quarantine) that has sufficient rescue equipments should be considered even if their results of RT-PCR test for pharyngeal swab specimens are negative to limit the spread of infection.

Therefore, the need for rapid bed-side diagnostic tools is highly appreciated. In this study we put for the first time a high sensitive score for suspicion of SARS-CoV-2 infection using bed-side investigation tools as chest X ray and lung ULS. A single clinician would be necessary to perform an initial medical assessment and imaging investigation directly at the patient's bed.

Lung ULS can help in reducing the number of health care professionals exposed during patient stratification by simple rapid bed side test (14)(15).

Lung ULS could be done without patient immobilization that might spread the infection. This differs from CT chest that requires patient mobilization and may be difficult in critical ventilated patients, in addition to its radiation hazards and high cost.

To the best of our knowledge, there is only one case report describing the findings of lung ULS in confirmed Covid 19 patient and reporting an irregular pleural line with small subpleural consolidations, areas of white lung and thick, confluents and irregular vertical artifacts (B-lines)(15).

Although our findings carry variable findings, however our results found that A profile and abnormal A lines in lung ULS are independent predictors for Covid 19 infection.

Our score has high sensitivity (93.8\%) in detection of Covid 19 infection making it a good negative test.

Lung ULS was reported to have $86 \%$ diagnostic accuracy in detecting alveolar consolidation and was able to differentiate between effusion and consolidation. Its specificity for detecting consolidation reached $100 \%$ in some studies (11).

Regarding the prognosis of Covid 19 infected patients, we analyzed the predictors for mortality as long as prolonged hospitalization. Old age, presence of cardiac problem and hypoxia on admission are clinical predictors of mortality. This is concordant with previous reports $(7)(16)$.

Concerning lung ULS, we present for the first time imaging predictors for mortality using this simple, safe and cheap tool in Covid 19 patients.

In our study B lung ULS profile and abnormal A lines were associated with mortality.

Abnormal A lines was also correlated with prolonged hospitalization. This also may direct the medical staff to determine patients needing high care and those that may need long hospital stay.

Regarding other investigation, CT chest shows more consolidation and ground glass appearance in Covid 19 infected patients. This is consistent with other reports in which the prominent radiologic abnormalities were bilateral

ground-glass opacity and subsegmental consolidation areas (8)(17).

Our laboratory findings showed that Covid 19 infected patients had less blood O2 saturation and less lymphocytic count than other causes of pneumonia. However, in our study lymphopenia has not reach the significant degree of correlation with mortality. Other reports showed predominance of lymphopenia in Covid 19 infection among critical than other less critical patients and is associated with severe course (7)(18). In a study conducted with Zhou et al, they found that baseline lymphocyte count was significantly higher in survivors than non-survivors of Covid 19 infected patients. However in survivors,

lymphocyte count improved after the 1st week of illness, whereas severe

lymphopenia remained until death in non-survivors(7). 


\section{Conclusion:}

Lung ULS could predict SARS-CoV-2 infection among patients with pulmonary symptoms necessitating admission. Lung ULS can also predict mortality in Covid 19 infected patients as well as their prolonged hospitalization.

\section{Recommendation:}

Lung ULS should be used to stratify the patients presented to ED with suspecting respiratory symptoms. The application of our clinical scoring system could exclude Covid 19 in those pneumonia patients considering that it is a good negative test.

\section{Limitations:}

The few number of patients are the main limitation of our study. Validation of our score is needed in subsequent similar studies.

Conflict of Interest: There is no conflict of interest concerning this study.

\section{References:}

1. Zhu N, Zhang D, Wang W, Li X, Yang B, Song J, et al. A novel coronavirus from patients with pneumonia in China, 2019. N Engl J Med. 2020;382(8):727-33.

2. Li Q, Guan X, Wu P, Wang X, Zhou L, Tong Y, et al. Early Transmission Dynamics in Wuhan, China, of Novel Coronavirus-Infected Pneumonia. N Engl J Med. 2020;382(13):1199-207.

3. Corman VM, Landt O, Kaiser M, Molenkamp R, Meijer A, Chu DKW, et al. Detection of 2019 novel coronavirus (2019-nCoV) by real-time RT-PCR. Eurosurveillance. 2020;25(3):1-8.

4. Li Y, Yao L, Li J, Chen L, Song Y, Cai Z, et al. Stability issues of RT-PCR testing of SARS-CoV-2 for hospitalized patients clinically diagnosed with COVID-19. J Med Virol. 2020;(March):1-6.

5. Shi H, Han X, Jiang N, Cao Y, Alwalid O, Gu J, et al. Radiological findings from 81 patients with COVID-19 pneumonia in Wuhan, China: a descriptive study. Lancet Infect Dis [Internet]. 2020;20(4):42534. Available from: http://dx.doi.org/10.1016/S1473-3099(20)30086-4

6. Wang YXJ, Liu W-H, Yang M, Chen W. The role of CT for Covid-19 patient's management remains poorly defined. Ann Transl Med. 2020;8(4):145-145.

7. Zhou F, Yu T, Du R, Fan G, Liu Y, Liu Z, et al. Clinical course and risk factors for mortality of adult inpatients with COVID-19 in Wuhan, China: a retrospective cohort study. Lancet [Internet]. 2020;395(10229):1054-62. Available from: http://dx.doi.org/10.1016/S0140-6736(20)30566-3

8. Guo W, Li M, Dong Y, Zhou H, Zhang Z, Tian C, et al. Diabetes is a risk factor for the progression and prognosis of COVID-19. Diabetes Metab Res Rev. 2020;(March):e3319.

9. Soldati G, Smargiassi A, Inchingolo R, Sher S, Nenna R, Valente S, et al. Lung ultrasonography may provide an indirect estimation of lung porosity and airspace geometry. Respiration. 2014;88(6):458-68.

10. Soldati G, Demi M, Smargiassi A, Inchingolo R, Demi L. The role of ultrasound lung artifacts in the diagnosis of respiratory diseases. Expert Rev Respir Med. 2019 Feb;13(2):163-72.

11. Danish M, Agarwal A, Goyal P, Gupta D, Lal H, Prasad R, et al. Diagnostic performance of 6-point lung ultrasound in ICU patients: A comparison with chest X-ray and CT thorax. Turkish J Anaesthesiol Reanim. 2019;47(4):307-19.

12. WHO Global Infection Prevention and Control Network. Infection prevention and control during health care when COVID-19 is suspected. 19/03/2020 [Internet]. 2020;(i):1-5. Available from: https://apps.who.int/iris/rest/bitstreams/1272420/retrieve 
13. Lichtenstein DA. BLUE-Protocol and FALLS-Protocol: Two Applications of Lung Ultrasound in the Critically Ill. Chest [Internet]. 2015 Jun 1;147(6):1659-70. Available from: https://doi.org/10.1378/chest.14-1313

14. Soldati G, Smargiassi A, Inchingolo R, Buonsenso D, Perrone T, Briganti DF, et al. Is there a role for lung ultrasound during the COVID-19 pandemic? J Ultrasound Med. 2020;1-4.

15. Buonsenso D, Piano A, Raffaelli F, Bonadia N, de Gaetano Donati K, Franceschi F. Point-of-Care Lung Ultrasound findings in novel coronavirus disease-19 pnemoniae: a case report and potential applications during COVID-19 outbreak. Eur Rev Med Pharmacol Sci. 2020;24(5):2776-80.

16. Ruan Q, Yang K, Wang W, Jiang L, Song J. Clinical predictors of mortality due to COVID-19 based on an analysis of data of 150 patients from Wuhan, China. Intensive Care Med [Internet]. 2020; Available from: https://doi.org/10.1007/s00134-020-05991-x

17. Chen N, Zhou M, Dong X, Qu J, Gong F, Han Y, et al. Epidemiological and clinical characteristics of 99 cases of 2019 novel coronavirus pneumonia in Wuhan, China: a descriptive study. Lancet [Internet]. 2020;395(10223):507-13. Available from: http://dx.doi.org/10.1016/S0140-6736(20)30211-7

18. Huang C, Wang Y, Li X, Ren L, Zhao J, Hu Y, et al. Clinical features of patients infected with 2019 novel coronavirus in Wuhan, China. Lancet. 2020;395(10223):497-506.

Legends:

Table 1:comparison between demographic and clinical data according to COVID19 test result.

Table 2:comparison between COVID19 positive and negative patients in; ABG, lympholytic count and some radiologic parameters

Table 3: Variables in the regression model.

Table 4: Four components of the score favoring the diagnosis of COVID19 positive patients if [?]5.

Table 5: showing the different parameters of the ROC analysis

Table 6: Variables associated with mortality in COVID19 positive patients.

Figure 1: ROC curve constructed to determine the cutoff value of the score with a sensitivity of $93.8 \%$ and a specificity of $58 \%$ in detecting COVID19 positive patients.

\section{Hosted file}

Figure 1.docx available at https://authorea.com/users/318929/articles/448758-new-score-todiagnose-covid-19-infection-using-lung-ultrasound 